\title{
Mitochondrial Dynamics and the ER: The Plant Perspective
}

\author{
Stefanie J. Mueller ${ }^{1}$ and Ralf Reski ${ }^{1,2,3 *}$ \\ ${ }^{1}$ Plant Biotechnology, Faculty of Biology, University of Freiburg, Freiburg, Germany, ${ }^{2}$ BIOSS Centre for Biological Signalling \\ Studies, University of Freiburg, Freiburg, Germany, ${ }^{3}$ FRIAS Freiburg Institute for Advanced Studies, University of Freiburg, \\ Freiburg, Germany, ${ }^{4}$ USIAS University of Strasbourg Institute for Advanced Study, University of Strasbourg, Strasbourg, \\ France
}

\section{OPEN ACCESS}

Edited by:

Michael Schrader,

University of Exeter, UK

Reviewed by:

Verena Kriechbaumer,

Sheffield Hallam University, UK

David C. Logan

Université d'Angers, France

*Correspondence:

Ralf Resk

ralf.reski@biologie.uni-freiburg.de

Specialty section

This article was submitted to Mitochondrial Research,

a section of the journal

Frontiers in Cell and Developmental

Biology

Received: 04 September 2015 Accepted: 23 November 2015 Published: 23 December 2015

Citation:

Mueller SJ and Reski R (2015) Mitochondrial Dynamics and the ER: The Plant Perspective.

Front. Cell Dev. Biol. 3:78. doi: 10.3389/fcell.2015.00078
Whereas contact sites between mitochondria and the ER have been in the focus of animal and fungal research for several years, the importance of this organellar interface and the molecular effectors are largely unknown for plants. This work gives an introduction into known evolutionary differences of molecular effectors of mitochondrial dynamics and interactions between animals, fungi, and plants. Using the model plant Physcomitrella patens, we provide microscopic evidence for the existence of mitochondria-ER interactions in plants and their correlation with mitochondrial constriction and fission. We further investigate a previously identified protein of unknown function (MELL1), and show that it modulates the amount of mitochondrial association to the ER, as well as mitochondrial shape and number.

Keywords: organelle dynamics, Physcomitrella patens, fission, fusion, Arabidopsis thaliana

\section{INTRODUCTION}

Subcellular compartmentation has enabled eukaryotes to simultaneously establish distinct reaction compartments with discrete protein content that need to be coordinated by interorganellar communication. Compartments are linked by signaling pathways and transport processes of different types of molecules such as proteins, lipids, and carbohydrates. Increasing evidence suggests that these processes are coordinated at specific contact interfaces (Prinz, 2014) which are either modulated by proteins or even membrane hemifusions (Mehrshahi et al., 2013, 2014). Multiple effectors of membrane contact sites (MCS) were identified linking the omnipresent ER to most other cell compartments, as e.g., the plasma membrane, lysosomes, vacuoles, and to mitochondria in mammals and yeast (for review, see Prinz, 2014). Identified functions of MCS include the transfer of lipids and the regulation of intracellular $\mathrm{Ca}^{2+}$ in animals and fungi (Prinz, 2014), and the accessibility to nonpolar metabolites between plant ER and plastids (Mehrshahi et al., 2014). In particular, the interactions between mitochondria and ER became a focus of research during the last decade in animals and fungi, linking ER-mitochondria contacts additionally to mitochondrial dynamics and quality control (Rowland and Voeltz, 2012; Kornmann, 2013; Lackner, 2014).

Mitochondria of a single cell have been described as a discontinuous whole (Logan, 2006), as they undergo frequent fusion and fission in animals, fungi, and plants (Arimura et al., 2004; Labbé et al., 2014), and thus maintain a certain rate of content exchange. This process was recently shown to be important for fatty acid metabolism in mammalian cells under starvation (Rambold et al., 2015), but is best known for its pivotal role in mitochondrial quality control (Twig et al., 2008b). Notably, mitochondrial fusion can either be transient ("kiss-and-run") while retaining mitochondrial identities, or of longer duration with increased exchange of matrix and also membrane content (Liu et al., 2009). 
A model for the mixing and unmixing of mitochondrial content was proposed, describing a separation of dysfunctional mitochondria from the pool of fusing mitochondria, and their targeting to autophagosomes (Twig et al., 2008b). In mammalian cells mitochondrial fusion triggers fission which in turn is followed by selective fusion (Twig et al., 2008b): differences in membrane potential become evident in daughter mitochondria after a fission event (Twig et al., 2008a). As membrane potential and import capacity are linked, the PINK/Parkin pathway subsequently regulates the exclusion of dysfunctional mitochondria via degradation of components of the fusion machinery in mammals (Narendra et al., 2012). A loss of this quality control system can in turn disturb stem cell fate in mammals (Katajisto et al., 2015) and leads to the decrease or the total loss of mitochondrial genomes in yeast and mammals (Labbé et al., 2014).

Although it was known for some time that mitochondrial form and function are linked, i.e., that changes in mitochondrial morphology and/or dynamics often are the first marker for cell stress in mammals, fungi, and plants (Scott and Logan, 2008; Welchen et al., 2014), the identity of several molecular effectors was only discovered in recent years. Thus, several components of the fission machinery are evolutionary conserved, such as dynamin-related GTPases (yeast Dnmlp, mammals Drp1, and A. thaliana DRP3A/DRP3B) and FIS-type proteins (FISSION, also called BIGYIN in plants; Scott and Logan, 2011). Notably, in both yeast and mammalian cells ER-mitochondrial contacts contribute to mitochondrial fission, supposedly either by the physical constriction of mitochondria by ER tubules, or as platforms for recruitment of the fission machinery (Friedman et al., 2011). In yeast, ER-mitochondrial interactions are mediated by the ERMES [ER-Mitochondrial Encounter Structure (Kornmann, 2013)] complex which has no known homologs in mammals or plants (Duncan et al., 2013; Kornmann, 2013).

The fusion machinery of mitochondria is largely conserved between mammals and yeast and involves the dynaminrelated GTPases homologous to the FUZZY ONIONS (Fzo) protein from Drosophila melanogaster: Fzolp in yeast and mitofusins (Mfn1, Mfn2) in mammals. These GTPases contain two C-terminal transmembrane domains and mediate tethering of neighboring organelles and outer membrane fusion (Labbé et al., 2014). In mammalian cells Mfn2/Mfn1 interactions additionally regulate mitochondrial/ER tethering and $\mathrm{Ca}^{2+}$ uptake (de Brito and Scorrano, 2008). In contrast, in land plants the closest homologs of this protein family localize to chloroplasts and mediate thylakoid architecture (Gao et al., 2006), raising the question how plant mitochondria fuse (Arimura et al., 2004; Scott and Logan, 2011).

Although evidence for links between plant mitochondrial form, function, and dynamics exist, the molecular identity of interaction sites is mostly unclear and modulators and effectors known from animal systems, such as Bcl2-like proteins, mitofusins, PINK, and Parkin (Logan, 2006, 2010; Elgass et al., 2013; Labbé et al., 2014) are lacking. Interestingly, plants possess a highly organized ER with different subdomains including potential contact sites to mitochondria, with suggested functional links to the transfer of lipids and mitochondrial dynamics (Staehelin, 1997; Sparkes et al., 2009; Stefano et al., 2014a).

We recently identified a plant protein with LEA (Late Embryogenesis Abundant) and LysM domains and a conspicuous subcellular localization to ER and mitochondria (MELL1), which influences mitochondrial shape (Mueller et al., 2014). Here, we describe its influence on the association between mitochondria and ER and discuss future challenges in mitochondrial dynamics research.

\section{RESULTS}

In order to monitor mitochondria and ER simultaneously in a plant, we used fluorescently labeled organelles of the model moss Physcomitrella patens, which provides a uniquely high rate of homologous recombination in plants (Strepp et al., 1998) and is amenable to confocal microscopy studies (Abel et al., 1989; Furt et al., 2012; Vidali and Bezanilla, 2012; Müller et al., 2015). We generated a stable transgenic moss line constitutively expressing mitochondria-targeted mEOS (mtEOS; Mathur et al., 2010) and transiently transfected protoplasts of this line with an ER marker that comprises a signal peptide, mCerulean, and a C-terminal KDEL ER retention signal (spCerKDEL). We found that mitochondria in moss protoplasts were mostly small elongated tubular structures which move only at about a 10th of the speed of flowering plant mitochondria (max. speed in our hands was $75 \mathrm{~nm} / \mathrm{s}$ ), which supports previous findings (Pressel et al., 2008; Furt et al., 2012). ER tubules tightly wrapped most mitochondria of a cell (Figure 1A). When investigating high quality images of mitochondria and ER $(n=51)$, a third of the mitochondria showed an elongated shape with clear constriction sites (Figure 1B). In our dataset, $88 \%$ of these constriction sites showed a clear co-localization with ER tubules. Mitochondrial constriction sites did not always lead to fission events in the time frame of several minutes. But when fission events occurred (Figure 1C), ER was closely associated and ER tubules remained attached on both newly generated ends of daughter mitochondria. Thus, ER and mitochondrial dynamics are linked in moss, although the causality of this correlation is as yet unclear.

As mitochondria and ER dynamics correlate, we further investigated whether ER-mitochondria association is altered by overexpression of the ER-mitochondria localized protein we recently identified (MELL1; Mueller et al., 2014). Figure 2A depicts $3 \mathrm{D}$ reconstructions of a typical protoplast expressing spCerKDEL in the mtmEOS background line (bg) or spCerKDEL and MELL1:GFP in the mtmEOS background line (ox). Mitochondrial shape is severely altered toward large mitochondria. Mitochondrial number is significantly reduced and sphericity of mitochondria significantly increased (Figure 2B), whereas the total volume of mitochondria was not significantly altered (Figure 2B). The increase in sphericity induces a trend toward decreased surface area of the mitochondria, which was not statistically significant in our dataset (Figure 2B). 


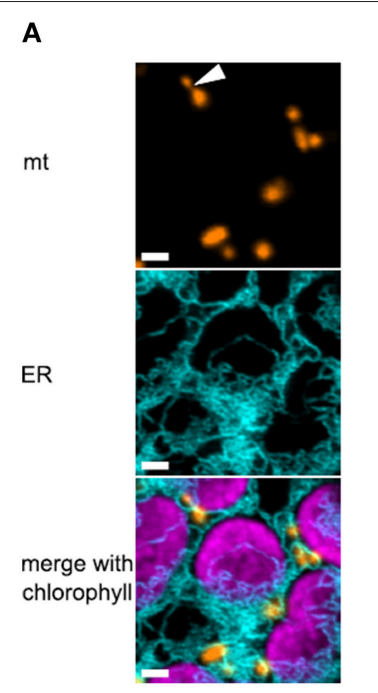

B

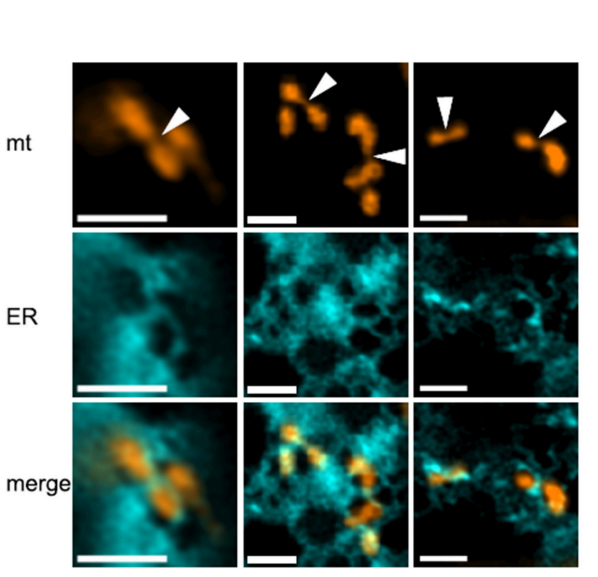

C

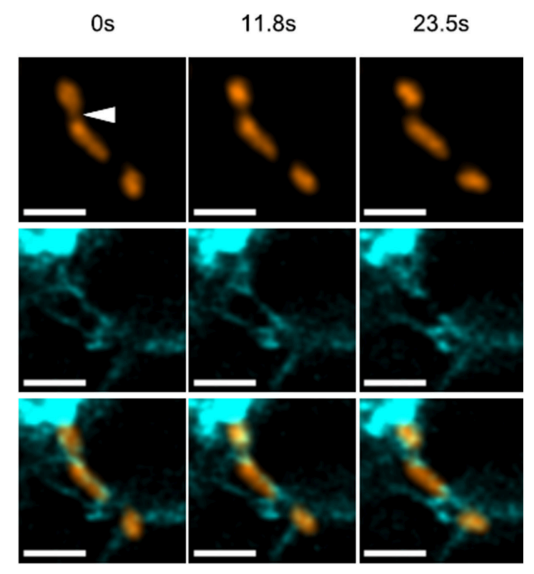

FIGURE 1 | Correlation between constriction sites in mitochondria and ER tubules in moss. (A) Overview of several mitochondria, the ER and chloroplasts in a protoplast of Physcomitrella patens. ER tubules are often closely associated to both organelles. The arrowhead points to a mitochondrion with a prominent constriction site, co-localizing with ER. Scale bars are $2 \mu \mathrm{m}$. (B) Several mitochondria show co-localization with the ER at constriction sites (arrowheads) and at their ends. Scale bars are $2 \mu \mathrm{m}$. (C) Time series ( $2 \mathrm{~min})$ of a mitochondrial fission event in moss, showing the close association of ER, which subsequently remains attached on both newly generated ends of daughter mitochondria. Scale bars are $2 \mu \mathrm{m}$.

Large mitochondria possess a common matrix space, as photobleaching of mtEOS lead to a concomitant decrease of fluorescence intensity in neighboring areas of the same mitochondrion (Figure 2C). We tracked the association of mitochondria and ER by Mander's co-localization coefficient (M1 Figure 2D) between different transfected cells (left), and over several time series (right, $\sim$ duration $2 \mathrm{~min}$ ). The co-localization of mitochondria with the ER was significantly increased for cells over-expressing MELL1, compared to cells of the background line. Moreover, the co-localization coefficient remained elevated during the time courses in MELL1 over-expressing cells, in contrast to a higher variance of mitochondria-ER co-localization in the background line. Thus, the association of mitochondria and ER is increased in MELL1 over-expressing cells and shows a high temporal persistency. Figure 2E depicts details of the association between mitochondria and ER under MELL1 overexpression. Mitochondria are embedded in a dense network of ER tubules and occasionally (Figure 2E arrowhead) show tubular extensions [matrixules (Logan, 2006)].

\section{DISCUSSION}

\section{Connectivity Between Organelles in Plant Cells}

In plants, the existence of specialized contact domains between the ER and other organelles such as chloroplasts was evidenced by several experimental approaches, either exerting mechanical forces by optical tweezers (Andersson et al., 2007), or using transorganellar complementation to demonstrate biochemical continuity (Mehrshahi et al., 2013). Mitochondria and ER cooperate in several biosynthetic pathways and exchange phospholipids in plants (for review see Millar et al., 2008). However, the molecular identity of proteins mediating contact sites and connectivity between mitochondria and ER in plants is so far unknown.

Studies investigating organelle movement in plants point to the presence of tethers or hemifused membranes between the ER and other organelles, as organelle dynamics correlate, without evidence for luminal connectivity (Stefano et al., 2014a,b). Two factors modulating these interactions may be membrane curvature and shape, as well as movement on cytoskeletal elements (Stefano et al., 2014a). Thus, inhibition of both actin filaments and microtubules was found to promote mitochondrial fusion in plants (Sheahan et al., 2005), probably indicating that movement on cytoskeletal elements counteracts complete fusion, similar to the situation in the mammalian system (Liu et al., 2009). Further, when the actin and the microtubule cytoskeleton were perturbed simultaneously, mitochondria tended to cluster around chloroplasts and ER patches (Van Gestel et al., 2002), implying mechanisms for specific association that lead to typical plant subcellular positioning (Welchen et al., 2014). As we have shown here, mitochondria co-localize with ER in moss protoplasts (Mander's coefficient $0.69 \pm 0.32$ ), often with one or several ER tubules crossing parts of the mitochondrial surface and the ends of elongated mitochondria. This colocalization showed a high variance in the mtEOS-labeled line, indicating frequent changes in the amount of ER in the ultimate proximity of mitochondria. Similar to animal and fungal model systems, we found that ER labeled mitochondria constriction sites, suggesting an evolutionary conservation of mitochondriaER interactions at constriction sites. In order to investigate the molecular basis and effect of this correlation, contact sites 


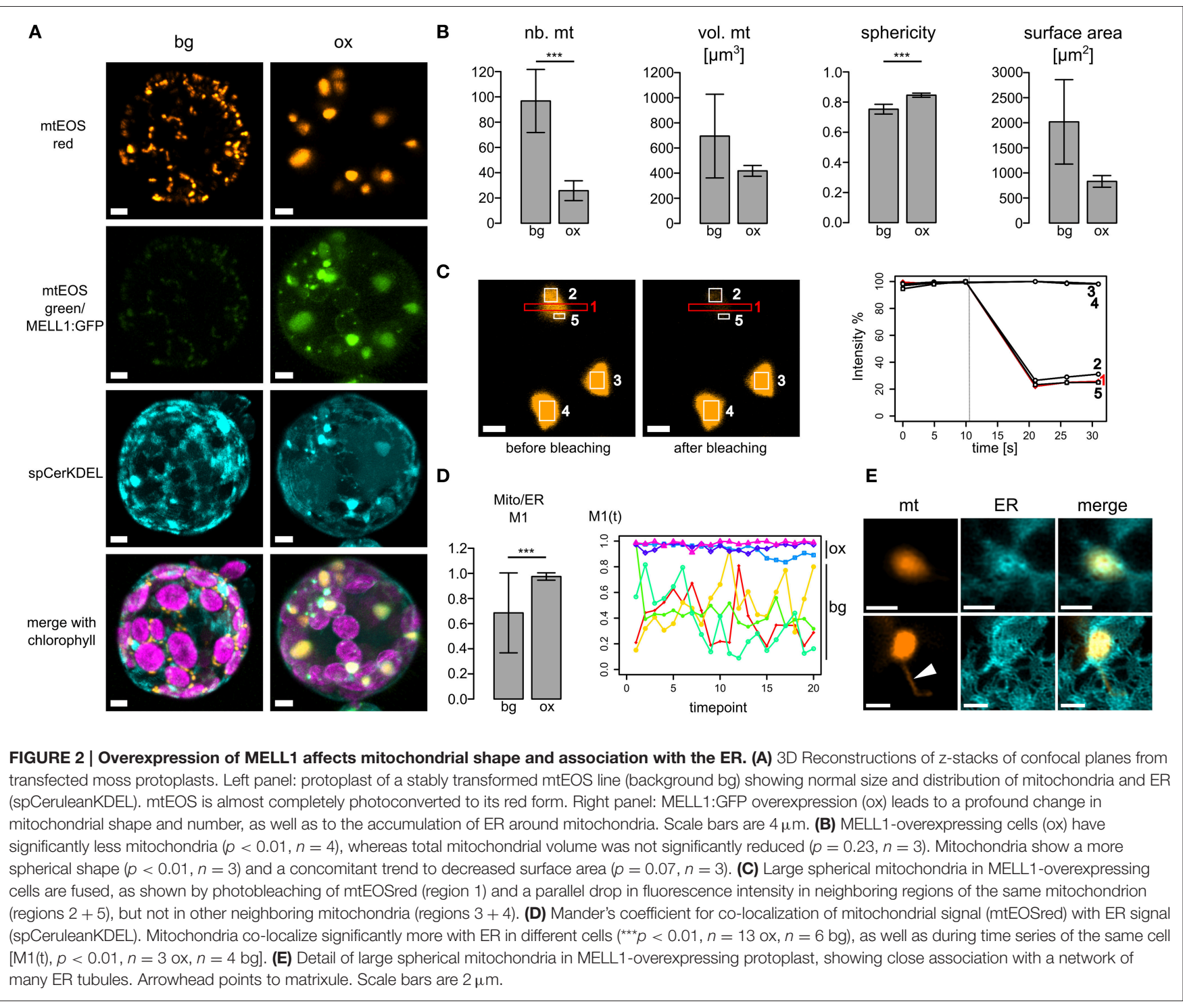

between ER and mitochondria in plants await identification, as no homologs to ERMES or mitofusins are present in plant mitochondria.

\section{MELL1 Level Influences the Association of Mitochondria to the ER}

In differentiated plant cells, mitochondria undergo frequent fusion and fission (Arimura et al., 2004) without global changes in number or shape, whereas differentiating protoplasts show massive mitochondrial fusions (Sheahan et al., 2005), putatively to redistribute mtDNA. Overexpression of MELL1 led to large fused mitochondria, which were closely associated to a constitutively high amount of ER. In theory, this phenotype could either relate to increased fusion of mitochondria, or decreased fission. Interestingly, though major changes in mitochondrial shape and distribution occurred, mitochondria were not dysfunctional, as indicated by correct targeting of the
mtEOS probe. Additionally, the ability to form tubular extensions (matrixules) was retained under MELL1 overexpression.

Using forward and reverse genetics, conserved molecular mechanisms behind mitochondrial fission as well as plantspecific modulators were characterized, such as NETWORK/ELM (ELongated Mitochondria) which is required for the localization of DRP3A to plant mitochondria (Arimura et al., 2008). In the model flowering plant Arabidopsis thaliana, the evolutionary conserved dynamin-related GTPases DRP3A and DRP3B mediate mitochondrial (and peroxisomal) fission (Fujimoto et al., 2009). Mutations of components of the fission machinery (DRP3A, DRP3B, FIS1A, FIS1B) lead to defects in mitochondrial shape and distribution, resulting in a reduced number of mitochondria with a more spherical shape (Scott et al., 2006; Zhang and $\mathrm{Hu}, 2008$; Fujimoto et al., 2009), similar to our results. Other plant mutants exhibiting an aggregation of mitochondria include FRIENDLY, a homolog to mammalian CLUH (clueless homolog; Gao et al., 2014), which causes 
clustering of mitochondria and an increase in matrix exchange, but no hyperfusion (El Zawily et al., 2014). CLUH was recently shown to bind mRNA of mitochondrially targeted proteins and may thus influence mitochondrial distribution indirectly via mitochondrial biogenesis (Gao et al., 2014). In MELL1 overexpressing protoplasts, mitochondria underwent complete fusion to large spherical mitochondria, with a common matrix space (Figure 2C) indicating a disturbed balance between fusion and fission. As this effect is accompanied by an increase in the association of ER to mitochondria, MELL1 might either directly or indirectly influence proteins at mitochondria-ER contact sites in plants. Whether MELL1 overexpression causes increased mitochondrial fusion or decreased mitochondrial fission is unclear so far. It is tempting to speculate that the increased mitochondrial association to the ER would disturb the fission machinery, as ER-mediated positional clues for fission, either provided by constriction via ER-tubules, or recruitment of the fission machinery to contact sites (Friedman et al., 2011), might be lacking. Alternatively, MELL1 might be a first link to the unknown mitochondrial fusion machinery in plants, although the protein does not contain a GTPase domain itself. An intriguing possibility is that MELL1 influences membrane curvature, as LEA domains may form alpha-helical structure which insert laterally into membranes (Tolleter et al., 2010; Candat et al., 2014). Future studies of knock-out mutants and mitochondrial dynamics in plants, as well as interacting proteins will address these open questions.

In conclusion, surprisingly little is known about the molecular identity of organelle contact sites in plants, but the evidence presented in this work points to an evolutionary conserved importance of mitochondrial dynamics and contacts to the ER between fungi, animals, and plants, while evolution may have shaped analogous molecular effectors. It will further be interesting to investigate whether there is any common mechanism in mitochondrial fusion shared by all eukaryotes. Future challenges include the identification of candidate proteins for organellar contact sites in plants, to further link changes in organellar form and function to the context of organelle connectivity, and to unravel the mechanisms behind balanced fusion/fission processes and quality control in mitochondria.

\section{MATERIAL AND METHODS}

\section{Cloning}

Mitochondria-targeted mEOS (Wiedenmann et al., 2004), containing the first $261 \mathrm{bp}$ of the Nicotiana plumbaginifolia mitochondrial ATP2-1 coding sequence (X02868) as N-terminal targeting signal (Logan and Leaver, 2000; Mathur et al., 2010), was amplified via PCR (F ATAAGTCGACATGGCTTCTCGG AGGCTTCT, R ATCCGAGCTCTTATCGTCTGGCATTG) and ligated via the introduced SalI and SacI restriction sites into a newly assembled vector backbone containing the moss Actin5 promoter (Weise et al., 2006) and a NOS terminator, as well as homologous regions for gene targeting to the " $P$. patens targeting site 2" (PTA2; Kubo et al., 2013) locus (pAct5_PTA2). To assemble this vector, PTA2 $5^{\prime}$ homologous region (F GCT
CTTCTCCTGGGGATTAATTATTGGAGG, R GAAAGAACG AATTCGATCGGATCCGCGACTAGTGAGAGAATGTT) and PTA2 $3^{\prime}$ homologous region (F CTAGTCGCGGATCCGAT CGAATTCGTTCTTTCTGTCATTAACTGG, R GCTCTTCAT TGTTCAGGATAATGGTTC) were amplified from genomic DNA, joined with two template PCR (Tian et al., 2004) and ligated into a pJET1.2 vector (Thermo Fisher Scientific). The expression cassette of Actin5 promoter, multiple cloning site, fluorescent protein, and NOS terminator (Mueller et al., 2014) was subsequently introduced between the PTA2 homologous regions with the restriction enzymes BamHI and EcoRI. To create an ER marker construct, the mCerulean coding sequence was amplified from $p G E M H E-X$-Cerulean (BIOSS toolbox Freiburg), and codons for the ER retention signal KDEL added to the Cterminus (F TACTGTCGACGTGAGCAAGGGCGAGGAG, R TTACAGCTCATCCTTCTTGTACAGCTCGTCCATGC). This construct was introduced in the pAct5_PTA2 via restriction and ligation using SalI and Ecl136II. Subsequently, the signal peptide from moss aspartic protease (Schaaf et al., 2004) was PCR amplified from genomic DNA (F ATCAGTCGACATGGGGGC ATCGAGGAGTGTT; R ATTAGTCGACGCGAGGGCTTGCC TCAGCTA) and introduced in front of the mCerulean::KDEL with SalI restriction and ligation.

\section{Moss Protoplast Transfection}

Moss protoplasts of the P. patens (Hedw.) Bruch \& Schimp. Gransden strain (International Moss Stock Center IMSC \#40001) were prepared and transfected as described previously (Strepp et al., 1998; Hohe et al., 2004; Mueller et al., 2014). For stable transformation, an uncut plasmid containing the nptII neomycin resistance cassette ( $p B S N N N E V)$ was co-transfected in a ratio of 3:1 with the construct for homologous recombination. For transient transfection, $p A c t 5 \_P T A 2$ vectors containing organelle marker constructs and MELL1 overexpression vector (Mueller et al., 2014) were used uncut (10 $\mu$ g per construct), whereas the construct was released from the vector creating homologous ends via BspQI restriction sites for stable transformation $(30 \mu \mathrm{g}$ used per transfection). Moss protoplasts were kept in the dark and imaged between 48 and $72 \mathrm{~h}$ after transfection. A stable mtEOS line (mtmEOS\#44) is available from the International Moss Stock Center (IMSC \#40776).

\section{Confocal Microcopy and Image Analysis}

All confocal images were taken with a Zeiss LSM 510 META with upright microscope Axio Imager Z1, using a C-Apochromat $63 \mathrm{x} / 1.2 \mathrm{~W}$ Korr objective with water immersion. Fluorophores were excited with either an Argon laser (3\% $488 \mathrm{~nm}$ for

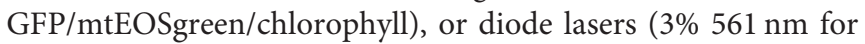
mtEOSred; 3\% $405 \mathrm{~nm}$ for Cerulean) using three separate tracks. Fluorescence was detected for chlorophyll from $670-756 \mathrm{~nm}$ (false colored magenta), for GFP from 505-550 nm (false colored green), for mtEOSred from 575-615 nm (false colored orange). Pinhole was set to $1 \mathrm{AU}$ for Cerulean channel and section thickness adjusted accordingly in all other channels. Pixel dwell was $1.61 \mu$ s. Images were taken using 4 averages and $256 \times 256$ pixel for time series ( $\sim 7 \mathrm{~s}$ per time point) and using 16 averages and $512 \times 512$ pixel for snaps. The zoom factor was adjusted 
to guarantee $1.5-2 \mathrm{x}$ overimaging of pixels, as recommended for deconvolution (see Huygens software manual). Bleaching (Figure 2C) settings for mtEOSred were used as follows: start after three scans, 300 iterations of bleaching, 100\% $561 \mathrm{~nm}$ laser.

Confocal images were all deconvolved prior to subsequent analyses using Huygens Remote Manager (v3.2.2, Scientific Volume Imaging; SNR $=8$ for time series, SNR $=10-15$ for snaps). Co-localization analysis was performed in Huygens using Mander's coefficient (Manders et al., 1993). Three-dimensional reconstructions of $\mathrm{z}$-stacks were performed after deconvolution using the Imaris software (Bitplane). Mitochondrial number was analyzed using the icy (http://icy.bioimageanalysis.org; de Chaumont et al., 2012) spot detector tool. Volume, shape, and surface area was analyzed by creating a surface from the mtEOSred channel in Imaris (Bitplane) and the surface statistics tool. Statistical analyses were conducted using the GraphPad Software Quickcalcs tools (http://www.graphpad.com/

\section{REFERENCES}

Abel, W. O., Knebel, W., Koop, H.-U., Marienfeld, J. R., Quader, H., Reski, R., et al. (1989). A cytokinin-sensitive mutant of the moss Physcomitrella patens defective in chloroplast division. Protoplasma 152, 1-13. doi: 10.1007/BF01354234

Andersson, M. X., Goksör, M., and Sandelius, A. S. (2007). Optical manipulation reveals strong attracting forces at membrane contact sites between endoplasmic reticulum and chloroplasts. J. Biol. Chem. 282, 1170-1174. doi: 10.1074/jbc.M608124200

Arimura, S., Fujimoto, M., Doniwa, Y., Kadoya, N., Nakazono, M., Sakamoto, W., et al. (2008). Arabidopsis ELONGATED MITOCHONDRIA1 is required for localization of DYNAMIN-RELATED PROTEIN3A to mitochondrial fission sites. Plant Cell 20, 1555-1566. doi: 10.1105/tpc.108. 058578

Arimura, S., Yamamoto, J., Aida, G. P., Nakazono, M., and Tsutsumi, N. (2004). Frequent fusion and fission of plant mitochondria with unequal nucleoid distribution. Proc. Natl. Acad. Sci. U.S.A. 101, 7805-7808. doi: 10.1073/pnas.0401077101

Candat, A., Paszkiewicz, G., Neveu, M., Gautier, R., Logan, D. C., AvelangeMacherel, M. H., et al. (2014). The ubiquitous distribution of late embryogenesis abundant proteins across cell compartments in Arabidopsis offers tailored protection against abiotic stress. Plant Cell 26, 3148-3166. doi: 10.1105/tpc.114.127316

de Brito, O. M., and Scorrano, L. (2008). Mitofusin 2 tethers endoplasmic reticulum to mitochondria. Nature 456, 605-610. doi: 10.1038/nature07534

de Chaumont, F., Dallongeville, S., Chenouard, N., Hervé, N., Pop, S., Provoost, T., et al. (2012). Icy: an open bioimage informatics platform for extended reproducible research. Nat. Methods 9, 690-696. doi: 10.1038/ nmeth. 2075

Duncan, O., Murcha, M. W., and Whelan, J. (2013). Unique components of the plant mitochondrial protein import apparatus. Biochim. Biophys. Acta 1833, 304-313. doi: 10.1016/j.bbamcr.2012.02.015

Elgass, K., Pakay, J., Ryan, M. T., and Palmer, C. S. (2013). Recent advances into the understanding of mitochondrial fission. Biochim. Biophys. Acta 1833, 150-161. doi: 10.1016/j.bbamcr.2012.05.002

El Zawily, A. M., Schwarzländer, M., Finkemeier, I., Johnston, I. G., Benamar, A., Cao, Y., et al. (2014). FRIENDLY regulates mitochondrial distribution, fusion, and quality control in Arabidopsis. Plant Physiol. 166, 808-828. doi: 10.1104/pp.114.243824

Friedman, J. R., Lackner, L. L., West, M., DiBenedetto, J. R., Nunnari, J., and Voeltz, G. K. (2011). ER tubules mark sites of mitochondrial division. Science 334, 358-362. doi: 10.1126/science. 1207385 quickcalcs/), using two-tailed T-test. Bar graphs show mean and standard deviation.

\section{AUTHOR CONTRIBUTIONS}

SM and RR planned and designed the research. SM performed experiments and analyzed data. SM and RR wrote the manuscript. All authors discussed data and approved the final version of the manuscript.

\section{ACKNOWLEDGMENTS}

Funding by the Excellence Initiative of the German Federal and State Governments (EXC294) is gratefully acknowledged. We are grateful to the team of the Life Imaging Center (LIC) at ZBSA Freiburg for their support. Further, we thank Richard Haas for technical support and Anne Katrin Prowse for proof-reading of the manuscript.

Fujimoto, M., Arimura, S., Mano, S., Kondo, M., Saito, C., Ueda, T., et al. (2009). Arabidopsis dynamin-related proteins DRP3A and DRP3B are functionally redundant in mitochondrial fission, but have distinct roles in peroxisomal fission. Plant J. Cell Mol. Biol. 58, 388-400. doi: 10.1111/j.1365313X.2009.03786.x

Furt, F., Lemoi, K., Tüzel, E., and Vidali, L. (2012). Quantitative analysis of organelle distribution and dynamics in Physcomitrella patens protonemal cells. BMC Plant Biol. 12:70. doi: 10.1186/1471-2229-12-70

Gao, H., Sage, T. L., and Osteryoung, K. W. (2006). FZL, an FZOlike protein in plants, is a determinant of thylakoid and chloroplast morphology. Proc. Natl. Acad. Sci. U.S.A. 103, 6759-6764. doi: 10.1073/pnas. 0507287103

Gao, J., Schatton, D., Martinelli, P., Hansen, H., Pla-Martin, D., Barth, E., et al. (2014). CLUH regulates mitochondrial biogenesis by binding mRNAs of nuclear-encoded mitochondrial proteins. J. Cell Biol. 207, 213-223. doi: $10.1083 /$ jcb. 201403129

Hohe, A., Egener, T., Lucht, J. M., Holtorf, H., Reinhard, C., Schween, G., et al. (2004). An improved and highly standardised transformation procedure allows efficient production of single and multiple targeted geneknockouts in a moss, Physcomitrella patens. Curr. Genet. 44, 339-347. doi: 10.1007/s00294-003-0458-4

Katajisto, P., Döhla, J., Chaffer, C. L., Pentinmikko, N., Marjanovic, N., Iqbal, S., et al. (2015). Asymmetric apportioning of aged mitochondria between daughter cells is required for stemness. Science 348, 340-343. doi: $10.1126 /$ science. 1260384

Kornmann, B. (2013). The molecular hug between the ER and the mitochondria. Curr. Opin. Cell Biol. 25, 443-448. doi: 10.1016/j.ceb.2013.02.010

Kubo, M., Imai, A., Nishiyama, T., Ishikawa, M., Sato, Y., Kurata, T., et al. (2013). System for stable $\beta$-Estradiol-inducible gene expression in the moss Physcomitrella patens. PLoS ONE 8:e77356. doi: 10.1371/journal.pone. 0077356

Labbé, K., Murley, A., and Nunnari, J. (2014). Determinants and functions of mitochondrial behavior. Annu. Rev. Cell Dev. Biol. 30, 357-391. doi: 10.1146/annurev-cellbio-101011-155756

Lackner, L. L. (2014). Shaping the dynamic mitochondrial network. BMC Biol. 12:35. doi: 10.1186/1741-7007-12-35

Liu, X., Weaver, D., Shirihai, O., and Hajnóczky, G. (2009). Mitochondrial "kiss-and-run": interplay between mitochondrial motility and fusion-fission dynamics. EMBO J. 28, 3074-3089. doi: 10.1038/emboj.2009.255

Logan, D. C. (2006). Plant mitochondrial dynamics. Biochim. Biophys. Acta 1763, 430-441. doi: 10.1016/j.bbamcr.2006.01.003

Logan, D. C. (2010). Mitochondrial fusion, division and positioning in plants. Biochem. Soc. Trans. 38, 789-795. doi: 10.1042/BST0380789 
Logan, D. C., and Leaver, C. J. (2000). Mitochondria-targeted GFP highlights the heterogeneity of mitochondrial shape, size and movement within living plant cells. J. Exp. Bot. 51, 865-871. doi: 10.1093/jexbot/ 51.346 .865

Manders, E. M. M., Verbeek, F. J., and Aten, J. A. (1993). Measurement of colocalization of objects in dual-colour confocal images. J. Microsc. 169, 375-382. doi: 10.1111/j.1365-2818.1993.tb03313.x

Mathur, J., Radhamony, R., Sinclair, A. M., Donoso, A., Dunn, N., Roach, E., et al. (2010). mEosFP-based green-to-red photoconvertible subcellular probes for plants. Plant Physiol. 154, 1573-1587. doi: 10.1104/pp.110.165431

Mehrshahi, P., Johnny, C., and DellaPenna, D. (2014). Redefining the metabolic continuity of chloroplasts and ER. Trends Plant Sci. 19, 501-507. doi: 10.1016/j.tplants.2014.02.013

Mehrshahi, P., Stefano, G., Andaloro, J. M., Brandizzi, F., Froehlich, J. E., and Dellapenna, D. (2013). Transorganellar complementation redefines the biochemical continuity of endoplasmic reticulum and chloroplasts. Proc. Natl. Acad. Sci. U.S.A. 110, 12126-12131. doi: 10.1073/pnas.1306331110

Millar, A. H., Small, I. D., Day, D. A., and Whelan, J. (2008). Mitochondrial biogenesis and function in Arabidopsis. Arab. Book Am. Soc. Plant Biol. 6:e0111. doi: 10.1199/tab.0111

Mueller, S. J., Lang, D., Hoernstein, S. N., Lang, E. G., Schuessele, C., Schmidt, A., et al. (2014). Quantitative analysis of the mitochondrial and plastid proteomes of the moss Physcomitrella patens reveals protein macrocompartmentation and microcompartmentation. Plant Physiol. 164, 2081-2095. doi: 10.1104/pp.114.235754

Müller, S. J., Gütle, D. D., Jacquot, J.-P., and Reski, R. (2015). Can mosses serve as model organisms for forest research? Ann. For. Sci. doi: 10.1007/s13595-0150468-7. [Epub ahead of print].

Narendra, D., Walker, J. E., and Youle, R. (2012). Mitochondrial quality control mediated by PINK1 and Parkin: Links to parkinsonism. Cold Spring Harb. Perspect. Biol. 4:a011338. doi: 10.1101/cshperspect.a011338

Pressel, S., Ligrone, R., and Duckett, J. G. (2008). Cellular differentiation in moss protonemata: a morphological and experimental study. Ann. Bot. 102, 227-245. doi: $10.1093 / \mathrm{aob} / \mathrm{mcn} 080$

Prinz, W. A. (2014). Bridging the gap: membrane contact sites in signaling, metabolism, and organelle dynamics. J. Cell Biol. 205, 759-769. doi: 10.1083/jcb.201401126

Rambold, A. S., Cohen, S., and Lippincott-Schwartz, J. (2015). Fatty acid trafficking in starved cells: regulation by lipid droplet lipolysis, autophagy, and mitochondrial fusion dynamics. Dev. Cell 32, 678-692. doi: 10.1016/j.devcel.2015.01.029

Rowland, A. A., and Voeltz, G. K. (2012). Endoplasmic reticulum-mitochondria contacts: function of the junction. Nat. Rev. Mol. Cell Biol. 13, 607-625. doi: $10.1038 / \mathrm{nrm} 3440$

Schaaf, A., Reski, R., and Decker, E. L. (2004). A novel aspartic proteinase is targeted to the secretory pathway and to the vacuole in the moss Physcomitrella patens. Eur. J. Cell Biol. 83, 145-152. doi: 10.1078/0171-9335-00371

Scott, I., and Logan, D. C. (2008). Mitochondrial morphology transition is an early indicator of subsequent cell death in Arabidopsis. New Phytol. 177, 90-101. doi: 10.1111/j.1469-8137.2007.02255.x

Scott, I., and Logan, D. C. (2011). "Mitochondrial dynamics" in Plant Mitochondria, ed F. Kempken (New York, NY: Springer New York), 31-63.

Scott, I., Tobin, A. K., and Logan, D. C. (2006). BIGYIN, an orthologue of human and yeast FIS1 genes functions in the control of mitochondrial size and number in Arabidopsis thaliana. J. Exp. Bot. 57, 1275-1280. doi: 10.1093/jxb/erj096

Sheahan, M. B., McCurdy, D. W., and Rose, R. J. (2005). Mitochondria as a connected population: ensuring continuity of the mitochondrial genome during plant cell dedifferentiation through massive mitochondrial fusion. Plant J. Cell Mol. Biol. 44, 744-755. doi: 10.1111/j.1365-313X.2005.02561.x
Sparkes, I. A., Frigerio, L., Tolley, N., and Hawes, C. (2009). The plant endoplasmic reticulum: a cell-wide web. Biochem. J. 423, 145-155. doi: 10.1042/BJ20091113

Staehelin, L. A. (1997). The plant ER: a dynamic organelle composed of a large number of discrete functional domains. Plant J. Cell Mol. Biol. 11, 1151-1165. doi: 10.1046/j.1365-313X.1997.11061151.x

Stefano, G., Hawes, C., and Brandizzi, F. (2014a). ER - the key to the highway. Curr. Opin. Plant Biol. 22C, 30-38. doi: 10.1016/j.pbi.2014.09.001

Stefano, G., Renna, L., and Brandizzi, F. (2014b). The endoplasmic reticulum exerts control over organelle streaming during cell expansion. J. Cell Sci. 127, 947-953. doi: $10.1242 /$ jcs. 139907

Strepp, R., Scholz, S., Kruse, S., Speth, V., and Reski, R. (1998). Plant nuclear gene knockout reveals a role in plastid division for the homolog of the bacterial cell division protein FtsZ, an ancestral tubulin. Proc. Natl. Acad. Sci. U.S.A. 95, 4368-4373. doi: 10.1073/pnas.95.8.4368

Tian, G. W., Mohanty, A., Chary, S. N., Li, S., Paap, B., Drakakaki, G., et al. (2004). High-throughput fluorescent tagging of full-length Arabidopsis gene products in planta. Plant Physiol. 135, 25-38. doi: 10.1104/pp.104.040139

Tolleter, D., Hincha, D. K., and Macherel, D. (2010). A mitochondrial late embryogenesis abundant protein stabilizes model membranes in the dry state. Biochim. Biophys. Acta 1798, 1926-1933. doi: 10.1016/j.bbamem.2010.06.029

Twig, G., Elorza, A., Molina, A. J. A., Mohamed, H., Wikstrom, J. D., Walzer, G., et al. (2008a). Fission and selective fusion govern mitochondrial segregation and elimination by autophagy. EMBO J. 27, 433-446. doi: 10.1038/sj.emboj.7601963

Twig, G., Hyde, B., and Shirihai, O. S. (2008b). Mitochondrial fusion, fission and autophagy as a quality control axis: the bioenergetic view. Biochim. Biophys. Acta 1777, 1092-1097. doi: 10.1016/j.bbabio.2008.05.001

Van Gestel, K., Köhler, R. H., and Verbelen, J. P. (2002). Plant mitochondria move on F-actin, but their positioning in the cortical cytoplasm depends on both F-actin and microtubules. J. Exp. Bot. 53, 659-667. doi: 10.1093/jexbot/53. 369.659

Vidali, L., and Bezanilla, M. (2012). Physcomitrella patens: a model for tip cell growth and differentiation. Curr. Opin. Plant Biol. 15, 625-631. doi: 10.1016/j.pbi.2012.09.008

Weise, A., Rodriguez-Franco, M., Timm, B., Hermann, M., Link, S., Jost, W., et al. (2006). Use of Physcomitrella patens actin $5^{\prime}$ regions for high transgene expression: Importance of $5^{\prime}$ introns. Appl. Microbiol. Biotechnol. 70, 337-345. doi: 10.1007/s00253-005-0087-6

Welchen, E., García, L., Mansilla, N., and Gonzalez, D. H. (2014). Coordination of plant mitochondrial biogenesis: keeping pace with cellular requirements. Front. Plant Sci. 4:551. doi: 10.3389/fpls.2013.00551

Wiedenmann, J., Ivanchenko, S., Oswald, F., Schmitt, F., Röcker, C., Salih, A., et al. (2004). EosFP, a fluorescent marker protein with UV-inducible green-to-red fluorescence conversion. Proc. Natl. Acad. Sci. U.S.A. 101, 15905-15910. doi: 10.1073/pnas.0403668101

Zhang, X. C., and Hu, J. P. (2008). FISSION1A and FISSION1B proteins mediate the fission of peroxisomes and mitochondria in Arabidopsis. Mol. Plant 1, 1036-1047. doi: $10.1093 / \mathrm{mp} / \mathrm{ssn} 056$

Conflict of Interest Statement: The authors declare that the research was conducted in the absence of any commercial or financial relationships that could be construed as a potential conflict of interest.

Copyright (c) 2015 Mueller and Reski. This is an open-access article distributed under the terms of the Creative Commons Attribution License (CC BY). The use, distribution or reproduction in other forums is permitted, provided the original author(s) or licensor are credited and that the original publication in this journal is cited, in accordance with accepted academic practice. No use, distribution or reproduction is permitted which does not comply with these terms. 